Supporting Information

"Mechanistic Study of 1,2-Dichloroethane Hydrodechlorination on $\mathrm{Cu}$-Rich Pt-Cu Alloys: Combining Reaction Kinetics Experiments with DFT Calculations and Microkinetic Modeling"

Lang Xu ${ }^{\mathrm{a}}$, Eric E. Stangland ${ }^{\mathrm{b}}$, James A. Dumesic ${ }^{\mathrm{a}}$, Manos Mavrikakis ${ }^{* a}$

a Department of Chemical \& Biological Engineering, University of Wisconsin-Madison, Madison, Wisconsin 53706, U.S.A.

${ }^{\mathrm{b}}$ Core Research and Development, Dow, Midland, MI 48667, U.S.A.

*Email: emavrikakis@wisc.edu

Number of pages: 11

Number of schemes: 1

Number of figures: 3

Number of tables: 4 


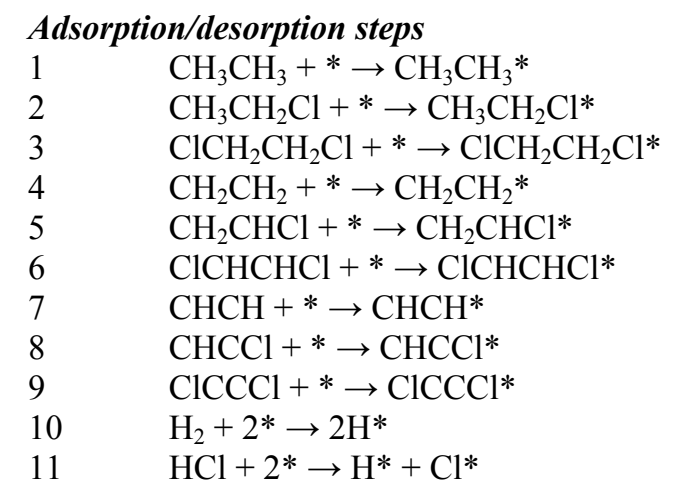

\section{Dehydrogenation/hydrogenation steps}

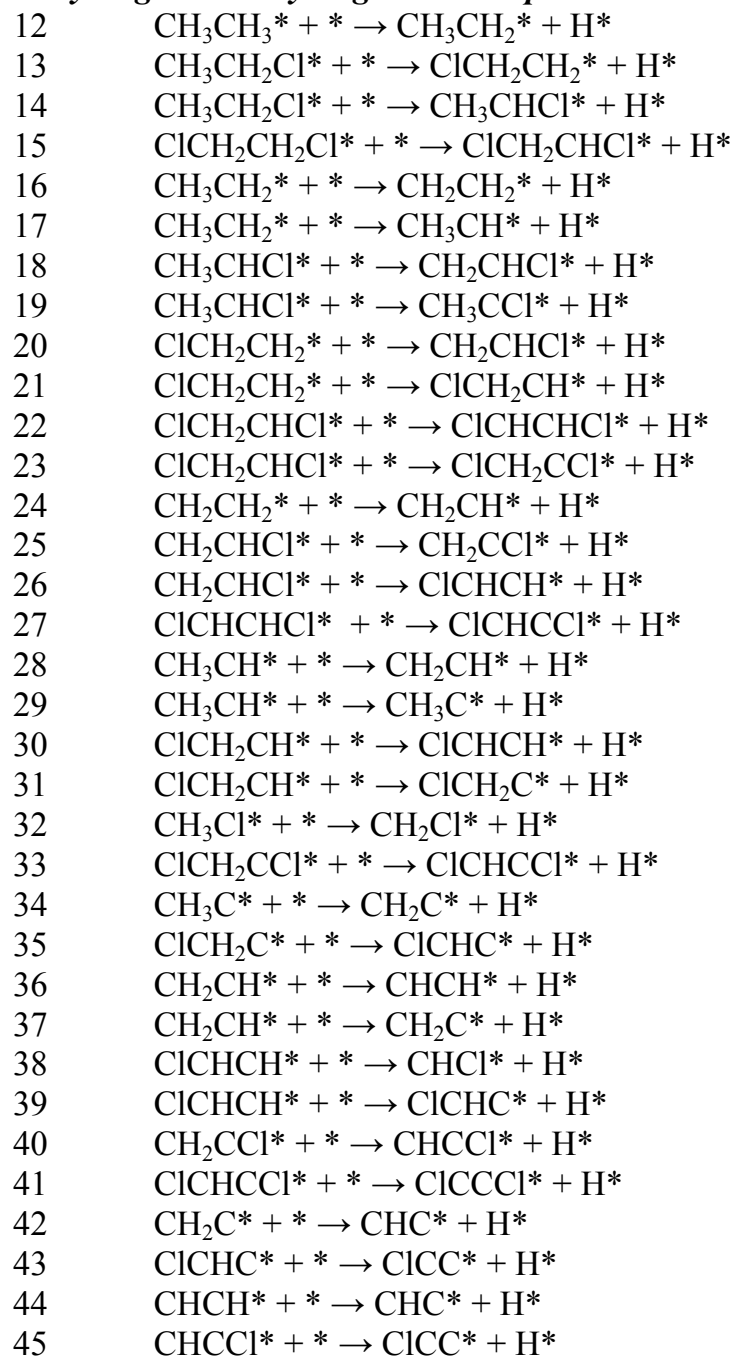

\section{Dechlorination/chlorination steps}

$\begin{array}{ll}46 & \mathrm{CH}_{3} \mathrm{CH}_{2} \mathrm{Cl}^{*}+* \rightarrow \mathrm{CH}_{3} \mathrm{CH}_{2} *+\mathrm{Cl}^{*} \\ 47 & \mathrm{ClCH}_{2} \mathrm{CH}_{2} \mathrm{Cl}^{*}+* \rightarrow \mathrm{ClCH}_{2} \mathrm{CH}_{2} *+\mathrm{Cl}^{*} \\ 48 & \mathrm{CH}_{3} \mathrm{CHCl}^{*}+* \rightarrow \mathrm{CH}_{3} \mathrm{CH}^{*}+\mathrm{Cl}^{*} \\ 49 & \mathrm{ClCH}_{2} \mathrm{CH}_{2}^{*}+* \rightarrow \mathrm{CH}_{2} \mathrm{CH}_{2} *+\mathrm{Cl}^{*} \\ 50 & \mathrm{ClCH}_{2} \mathrm{CHCl}^{*}+* \rightarrow \mathrm{CH}_{2} \mathrm{CHCl}^{*}+\mathrm{Cl}^{*} \\ 51 & \mathrm{ClCH}_{2} \mathrm{CHCl}^{*}+* \rightarrow \mathrm{ClCH}_{2} \mathrm{CH}^{*}+\mathrm{Cl}^{*}\end{array}$




$\begin{array}{ll}52 & \mathrm{CH}_{2} \mathrm{CHCl}^{*}+* \rightarrow \mathrm{CH}_{2} \mathrm{CH}^{*}+\mathrm{Cl}^{*} \\ 53 & \mathrm{ClCHCHCl}^{*}+\rightarrow \mathrm{ClCHCH}^{*}+\mathrm{Cl}^{*} \\ 54 & \mathrm{ClCH}_{2} \mathrm{CH}^{*}+* \rightarrow \mathrm{CH}_{2} \mathrm{CH}^{*}+\mathrm{Cl}^{*} \\ 55 & \mathrm{CH}_{3} \mathrm{CCl}^{*}+* \rightarrow \mathrm{CH}_{3} \mathrm{C}^{*}+\mathrm{Cl}^{*} \\ 56 & \mathrm{ClCH}_{2} \mathrm{CCl}^{*}+* \rightarrow \mathrm{CH}_{2} \mathrm{CCl}^{*}+\mathrm{Cl}^{*} \\ 57 & \mathrm{ClCH}_{2} \mathrm{CCl}^{*}+\rightarrow \mathrm{ClCH}_{2} \mathrm{C}^{*}+\mathrm{Cl}^{*} \\ 58 & \mathrm{ClCH}_{2} \mathrm{C}^{*}+\rightarrow \mathrm{CH}_{2} \mathrm{C}^{*}+\mathrm{Cl}^{*} \\ 59 & \mathrm{ClCHCH}^{*}+* \rightarrow \mathrm{CHCH}^{*}+\mathrm{Cl}^{*} \\ 60 & \mathrm{CH}_{2} \mathrm{CCl}^{*}+\rightarrow \mathrm{CH}_{2} \mathrm{C}^{*}+\mathrm{Cl}^{*} \\ 61 & \mathrm{ClCHCCl}^{*}+\rightarrow \mathrm{CHCCl}^{*} \mathrm{Cl}^{*} \\ 62 & \mathrm{ClCHCCl}^{*}+\rightarrow \mathrm{ClCHC}^{*}+\mathrm{Cl}^{*} \\ 63 & \mathrm{ClCHC}^{*}+\rightarrow \mathrm{CHC}^{*}+\mathrm{Cl}^{*} \\ 64 & \mathrm{CHCCl}^{*}+* \rightarrow \mathrm{CHC}^{*}+\mathrm{Cl}^{*} \\ 65 & \mathrm{ClCCCl}^{*}+* \rightarrow \mathrm{ClCC}^{*}+\mathrm{Cl}^{*}\end{array}$

Scheme S1: List of elementary steps considered for 1,2-DCA hydrodechlorination. This is the same reaction network as previously adopted in reference [1]. All the elementary steps are written in the bond-breaking direction for consistency. During the actual reaction, some steps might occur in the reverse direction (e.g., the hydrogenation of $\mathrm{CH}_{2} \mathrm{CH}_{2} *$ to $\mathrm{CH}_{3} \mathrm{CH}_{2}{ }^{*}$, the reverse of Step 16). 

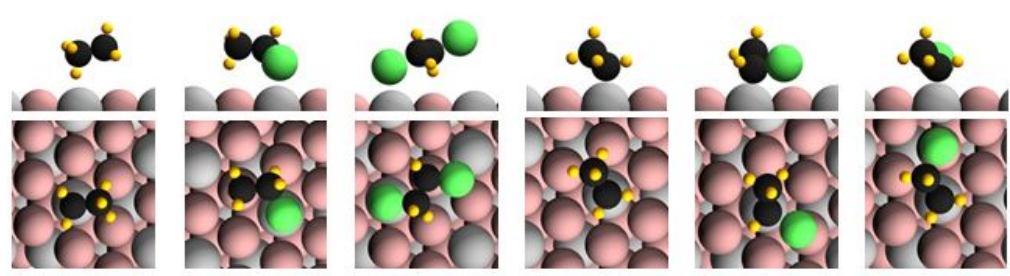

$\mathrm{CH}_{3} \mathrm{CH}_{3}$

$\mathrm{CH}_{3} \mathrm{CH}_{2} \mathrm{Cl}$

$\mathrm{ClCH}_{2} \mathrm{CH}_{2} \mathrm{Cl}$

$\mathrm{CH}_{3} \mathrm{CH}_{2}$

$\mathrm{CH}_{3} \mathrm{CHCl}$

$\mathrm{ClCH}_{2} \mathrm{CH}_{2}$
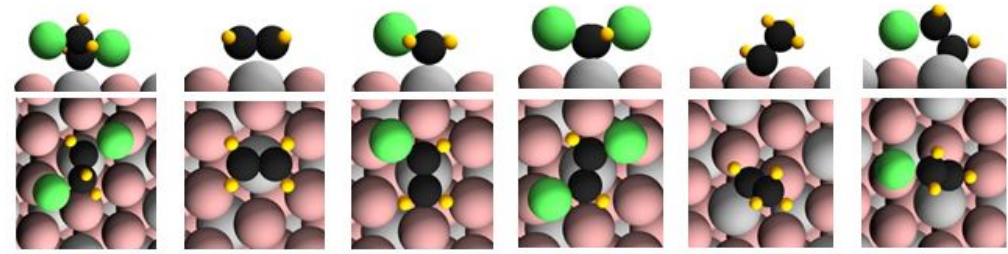

$\mathrm{ClCH}_{2} \mathrm{CHCl}$

$\mathrm{CH}_{2} \mathrm{CH}_{2}$

$\mathrm{CH}_{2} \mathrm{CHCl}$

$\mathrm{ClCHCHCl}$

$\mathrm{CH}_{3} \mathrm{CH}$

$\mathrm{ClCH}_{2} \mathrm{CH}$
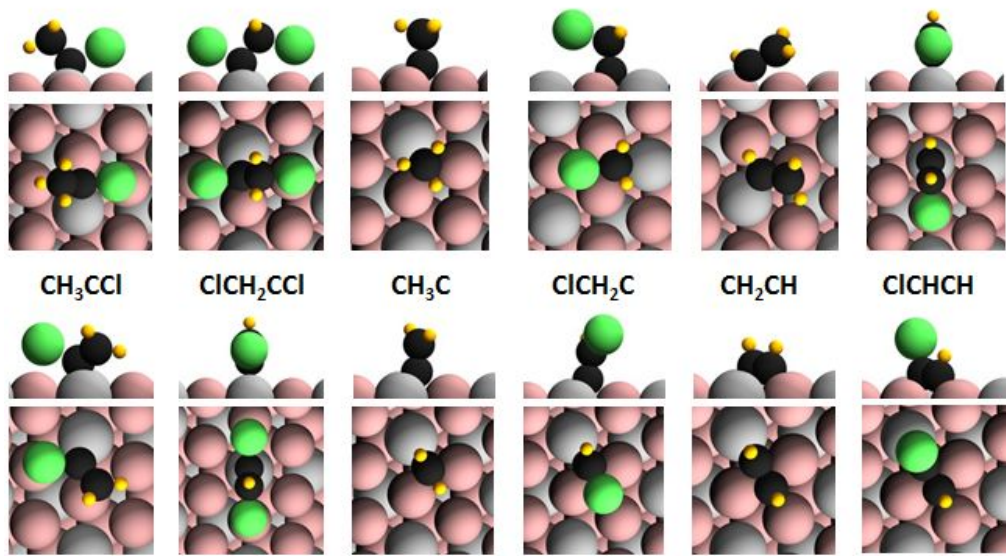

$\mathrm{ClCH}_{2} \mathrm{CCl}$

$\mathrm{CH}_{3} \mathrm{C}$

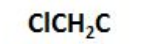

$\mathrm{CH}_{2} \mathrm{CH}$

$\mathrm{ClCHCH}$
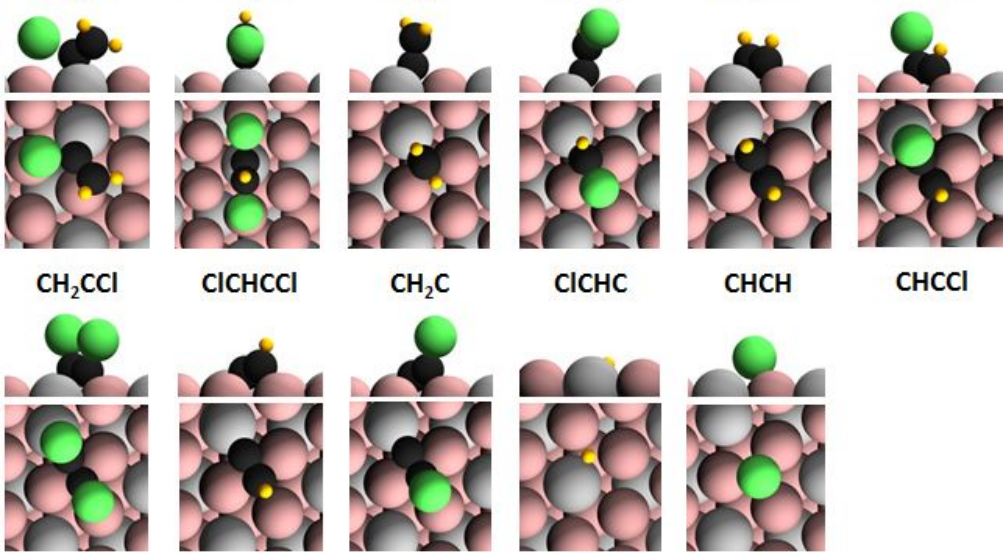

CICHCC

$$
\mathrm{CH}_{2} \mathrm{C}
$$

$\mathrm{ClCHC}$

$\mathrm{CHCH}$

$\mathrm{CHCCl}$
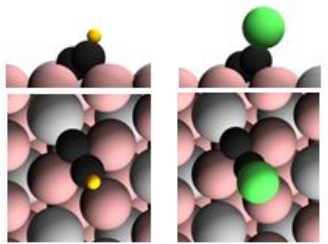

CHC

CICC

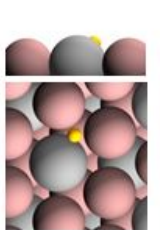

H

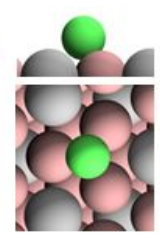

Cl

Figure S1: Cross-section and top views of minimum-energy structures of 1,2-DCA hydrodechlorination reaction intermediates adsorbed on $\mathrm{Pt}_{1} \mathrm{Cu}_{3}(111)$. Color code: grey $-\mathrm{Pt}$; pink $-\mathrm{Cu}$; black $-\mathrm{C}$; green $-\mathrm{Cl}$; yellow $-\mathrm{H}$. 

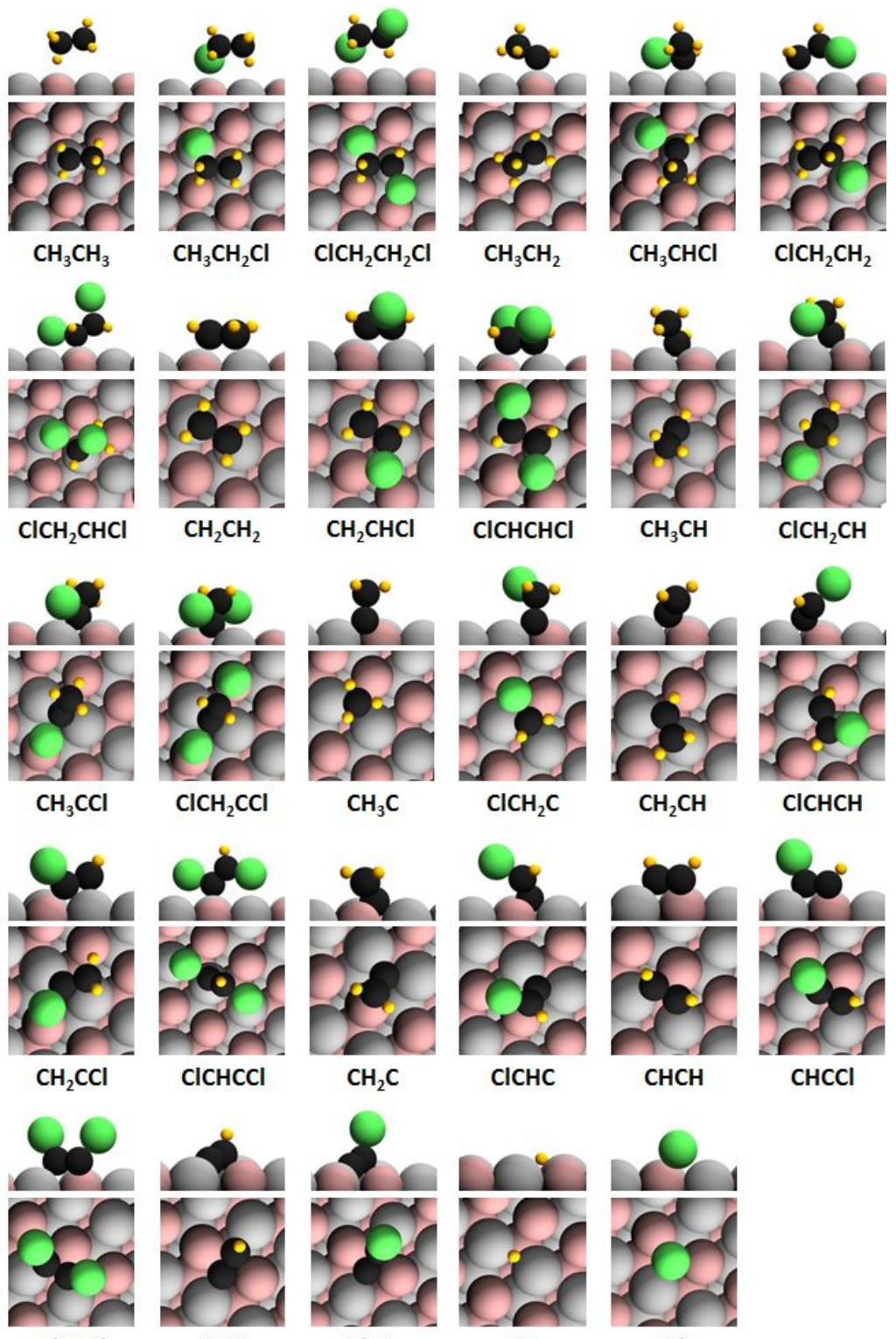

CICC
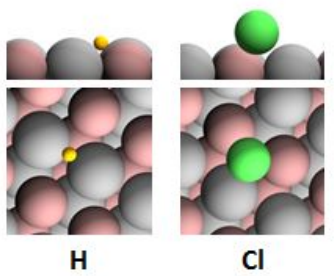

Figure S2: Cross-section and top views of minimum-energy structures of 1,2-DCA hydrodechlorination reaction intermediates adsorbed on $\mathrm{Pt}_{1} \mathrm{Cu}_{1}(111)$. Color code: grey $-\mathrm{Pt}$; pink $-\mathrm{Cu}$; black $-\mathrm{C}$; green $-\mathrm{Cl}$; yellow $-\mathrm{H}$. 

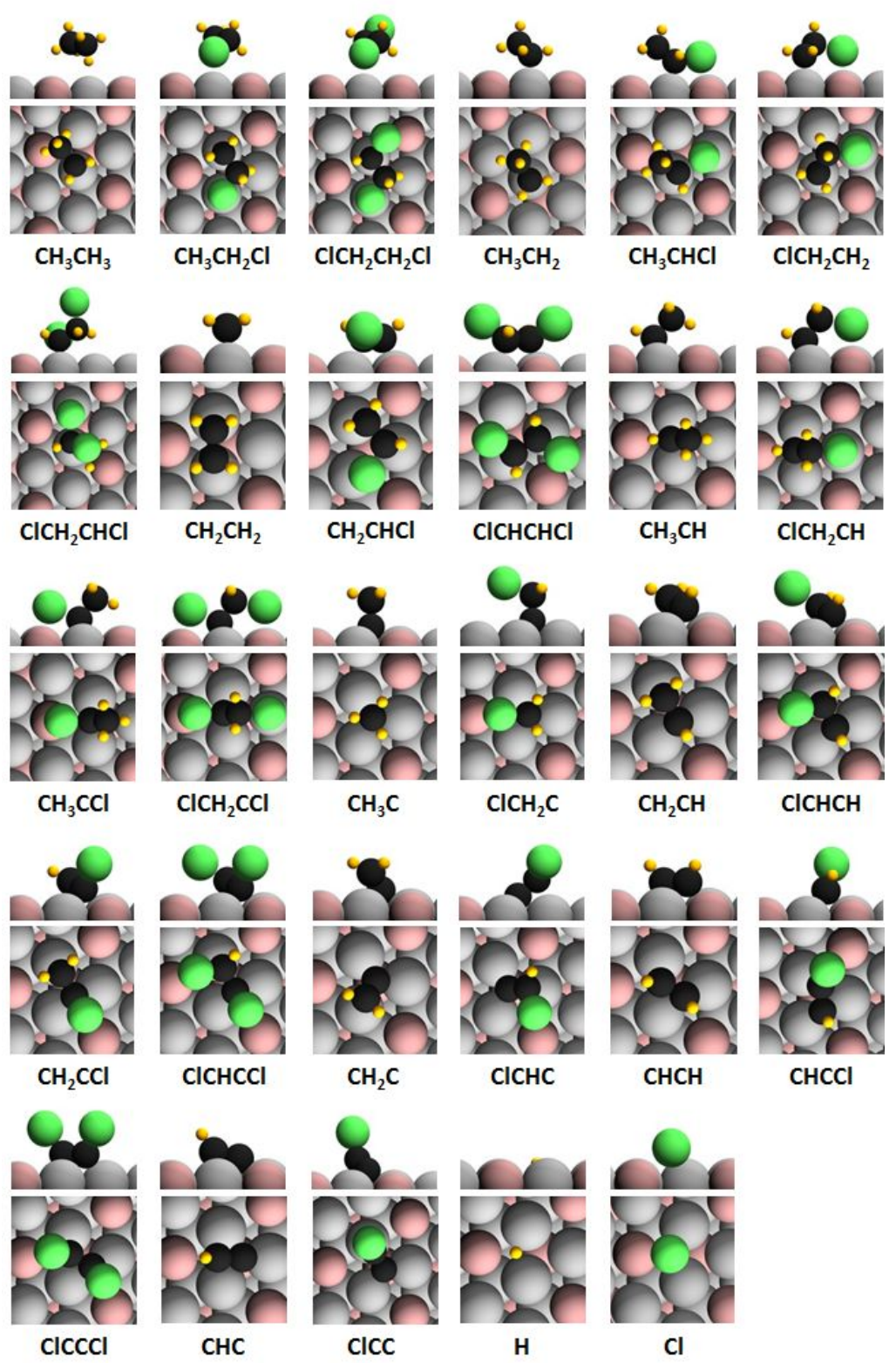

Figure S3: Cross-section and top views of minimum-energy structures of 1,2-DCA hydrodechlorination reaction intermediates adsorbed on $\mathrm{Pt}_{3} \mathrm{Cu}_{1}(111)$. Color code: grey $-\mathrm{Pt}$; pink $-\mathrm{Cu}$; black $-\mathrm{C}$; green $-\mathrm{Cl}$; yellow $-\mathrm{H}$. 
Table S1: Reaction energies $(\Delta E)$ and activation energy barriers $\left(E_{a}\right.$ and $E_{a, r e v}$, for forward and reverse reactions, respectively) of elementary steps for 1,2-DCA hydrodechlorination on $\mathrm{Pt}_{1} \mathrm{Cu}_{1}(111)$

\begin{tabular}{|c|c|c|c|c|}
\hline \# & Step & $\Delta E(\mathrm{eV})^{\mathrm{a}}$ & $E_{a}(\mathrm{eV})^{\mathrm{a}}$ & $E_{a, r e v}(e V)^{\mathrm{a}}$ \\
\hline 1 & $\mathrm{CH}_{3} \mathrm{CH}_{3}+* \rightarrow \mathrm{CH}_{3} \mathrm{CH}_{3} *$ & -0.37 & 0 & 0.37 \\
\hline 2 & $\mathrm{CH}_{3} \mathrm{CH}_{2} \mathrm{Cl}+* \rightarrow \mathrm{CH}_{3} \mathrm{CH}_{2} \mathrm{Cl}^{*}$ & -0.71 & 0 & 0.71 \\
\hline 3 & $\mathrm{ClCH}_{2} \mathrm{CH}_{2} \mathrm{Cl}+* \rightarrow \mathrm{ClCH}_{2} \mathrm{CH}_{2} \mathrm{Cl}^{*}$ & -0.76 & 0 & 0.76 \\
\hline 4 & $\mathrm{CH}_{2} \mathrm{CH}_{2}+* \rightarrow \mathrm{CH}_{2} \mathrm{CH}_{2} *$ & -1.34 & 0 & 1.34 \\
\hline 5 & $\mathrm{CH}_{2} \mathrm{CHCl}+* \rightarrow \mathrm{CH}_{2} \mathrm{CHCl}^{*}$ & -1.41 & 0 & 1.41 \\
\hline 6 & $\mathrm{ClCHCHCl}+* \rightarrow \mathrm{ClCHCHCl}^{*}$ & -1.51 & 0 & 1.51 \\
\hline 7 & $\mathrm{CHCH}+* \rightarrow \mathrm{CHCH}^{*}$ & -2.27 & 0 & 2.27 \\
\hline 8 & $\mathrm{CHCCl}+* \rightarrow \mathrm{CHCCl}^{*}$ & -2.57 & 0 & 2.57 \\
\hline 9 & $\mathrm{ClCCCl}+* \rightarrow \mathrm{ClCCCl}^{*}$ & -2.88 & 0 & 2.88 \\
\hline 10 & $\mathrm{H}_{2}+2 * \rightarrow 2 \mathrm{H}^{*}$ & -1.07 & 0 & 1.07 \\
\hline 11 & $\mathrm{HCl}+2^{*} \rightarrow \mathrm{H}^{*}+\mathrm{Cl}^{*}$ & -1.40 & 0 & 1.40 \\
\hline 12 & $\mathrm{CH}_{3} \mathrm{CH}_{3}^{*}+* \rightarrow \mathrm{CH}_{3} \mathrm{CH}_{2}^{*}+\mathrm{H}^{*}$ & 0.00 & 0.72 & 0.72 \\
\hline 13 & $\mathrm{CH}_{3} \mathrm{CH}_{2} \mathrm{Cl}^{*}+* \rightarrow \mathrm{ClCH}_{2} \mathrm{CH}_{2} *+\mathrm{H}^{*}$ & 0.05 & 0.86 & 0.81 \\
\hline 14 & $\mathrm{CH}_{3} \mathrm{CH}_{2} \mathrm{Cl}^{*}+* \rightarrow \mathrm{CH}_{3} \mathrm{CHCl}^{*}+\mathrm{H}^{*}$ & -0.12 & 1.09 & 1.21 \\
\hline 15 & $\mathrm{ClCH}_{2} \mathrm{CH}_{2} \mathrm{Cl}^{*}+* \rightarrow \mathrm{ClCH}_{2} \mathrm{CHCl}^{*}+\mathrm{H}^{*}$ & -0.18 & 1.06 & 1.23 \\
\hline 16 & $\mathrm{CH}_{3} \mathrm{CH}_{2} *+* \rightarrow \mathrm{CH}_{2} \mathrm{CH}_{2}^{*}+\mathrm{H}^{*}$ & -0.27 & 0.58 & 0.85 \\
\hline 17 & $\mathrm{CH}_{3} \mathrm{CH}_{2}{ }^{*}+* \rightarrow \mathrm{CH}_{3} \mathrm{CH}^{*}+\mathrm{H}^{*}$ & -0.13 & 0.56 & 0.69 \\
\hline 18 & $\mathrm{CH}_{3} \mathrm{CHCl}^{*}+* \rightarrow \mathrm{CH}_{2} \mathrm{CHCl}^{*}+\mathrm{H}^{*}$ & 0.03 & 1.19 & 1.16 \\
\hline 19 & $\mathrm{CH}_{3} \mathrm{CHCl}^{*}+* \rightarrow \mathrm{CH}_{3} \mathrm{CCl}^{*}+\mathrm{H}^{*}$ & -0.03 & 1.01 & 1.04 \\
\hline 20 & $\mathrm{ClCH}_{2} \mathrm{CH}_{2} *+* \rightarrow \mathrm{CH}_{2} \mathrm{CHCl}^{*}+\mathrm{H}^{*}$ & -0.14 & 0.98 & 1.13 \\
\hline 21 & $\mathrm{ClCH}_{2} \mathrm{CH}_{2}{ }^{*}+* \rightarrow \mathrm{ClCH}_{2} \mathrm{CH}^{*}+\mathrm{H}^{*}$ & 0.01 & 0.78 & 0.76 \\
\hline 22 & $\mathrm{ClCH}_{2} \mathrm{CHCl}^{*}+* \rightarrow \mathrm{ClCHCHCl}^{*}+\mathrm{H}^{*}$ & -0.01 & 1.30 & 1.31 \\
\hline 23 & $\mathrm{ClCH}_{2} \mathrm{CHCl}^{*}+* \rightarrow \mathrm{ClCH}_{2} \mathrm{CCl}^{*}+\mathrm{H}^{*}$ & -0.01 & 1.20 & 1.21 \\
\hline 24 & $\mathrm{CH}_{2} \mathrm{CH}_{2}^{*}+* \rightarrow \mathrm{CH}_{2} \mathrm{CH}^{*}+\mathrm{H}^{*}$ & 0.34 & 0.84 & 0.49 \\
\hline 25 & $\mathrm{CH}_{2} \mathrm{CHCl}^{*}+* \rightarrow \mathrm{CH}_{2} \mathrm{CCl}^{*}+\mathrm{H}^{*}$ & 0.31 & 1.04 & 0.74 \\
\hline 26 & $\mathrm{CH}_{2} \mathrm{CHCl}^{*}+* \rightarrow \mathrm{ClCHCH}^{*}+\mathrm{H}^{*}$ & 0.35 & 0.96 & 0.61 \\
\hline 27 & $\mathrm{ClCHCHCl}^{*}+* \rightarrow \mathrm{ClCHCCl}^{*}+\mathrm{H}^{*}$ & 0.40 & 1.20 & 0.80 \\
\hline 28 & $\mathrm{CH}_{3} \mathrm{CH}^{*}+* \rightarrow \mathrm{CH}_{2} \mathrm{CH}^{*}+\mathrm{H}^{*}$ & 0.20 & 0.93 & 0.73 \\
\hline 29 & $\mathrm{CH}_{3} \mathrm{CH}^{*}+* \rightarrow \mathrm{CH}_{3} \mathrm{C}^{*}+\mathrm{H}^{*}$ & -0.34 & 0.76 & 1.10 \\
\hline 30 & $\mathrm{ClCH}_{2} \mathrm{CH}^{*}+* \rightarrow \mathrm{ClCHCH}^{*}+\mathrm{H}^{*}$ & 0.19 & 1.03 & 0.84 \\
\hline 31 & $\mathrm{ClCH}_{2} \mathrm{CH}^{*}+* \rightarrow \mathrm{ClCH}_{2} \mathrm{C}^{*}+\mathrm{H}^{*}$ & -0.13 & 1.01 & 1.15 \\
\hline 32 & $\mathrm{CH}_{3} \mathrm{Cl}^{*}+* \rightarrow \mathrm{CH}_{2} \mathrm{Cl}^{*}+\mathrm{H}^{*}$ & 0.36 & 1.15 & 0.79 \\
\hline 33 & $\mathrm{ClCH}_{2} \mathrm{CCl}^{*}+* \rightarrow \mathrm{ClCHCCl}^{*}+\mathrm{H}^{*}$ & 0.40 & 1.20 & 0.80 \\
\hline 34 & $\mathrm{CH}_{3} \mathrm{C}^{*}+* \rightarrow \mathrm{CH}_{2} \mathrm{C}^{*}+\mathrm{H}^{*}$ & 0.25 & 1.13 & 0.88 \\
\hline 35 & $\mathrm{ClCH}_{2} \mathrm{C}^{*}+* \rightarrow \mathrm{ClCHC}^{*}+\mathrm{H}^{*}$ & 0.12 & 1.06 & 0.94 \\
\hline 36 & $\mathrm{CH}_{2} \mathrm{CH}^{*}+* \rightarrow \mathrm{CHCH}^{*}+\mathrm{H}^{*}$ & -0.07 & 0.64 & 0.71 \\
\hline 37 & $\mathrm{CH}_{2} \mathrm{CH}^{*}+* \rightarrow \mathrm{CH}_{2} \mathrm{C}^{*}+\mathrm{H}^{*}$ & -0.30 & 0.47 & 0.77 \\
\hline 38 & $\mathrm{ClCHCH}^{*}+* \rightarrow \mathrm{CHCl}^{*}+\mathrm{H}^{*}$ & -0.10 & 0.74 & 0.83 \\
\hline 39 & $\mathrm{ClCHCH}^{*}+* \rightarrow \mathrm{ClCHC}^{*}+\mathrm{H}^{*}$ & -0.21 & 1.12 & 1.33 \\
\hline 40 & $\mathrm{CH}_{2} \mathrm{CCl}^{*}+* \rightarrow \mathrm{CHCCl}^{*}+\mathrm{H}^{*}$ & -0.06 & 0.85 & 0.91 \\
\hline 41 & $\mathrm{ClCHCCl}^{*}+* \rightarrow \mathrm{ClCCCl}^{*}+\mathrm{H}^{*}$ & -0.22 & 0.61 & 0.84 \\
\hline 42 & $\mathrm{CH}_{2} \mathrm{C}^{*}+* \rightarrow \mathrm{CHC}^{*}+\mathrm{H}^{*}$ & 0.57 & 1.61 & 1.04 \\
\hline 43 & $\mathrm{ClCHC}^{*}+* \rightarrow \mathrm{ClCC}^{*}+\mathrm{H}^{*}$ & 0.63 & 1.59 & 0.96 \\
\hline 44 & $\mathrm{CHCH}^{*}+* \rightarrow \mathrm{CHC}^{*}+\mathrm{H}^{*}$ & 0.34 & 1.15 & 0.81 \\
\hline 45 & $\mathrm{CHCCl}^{*}+* \rightarrow \mathrm{ClCC}^{*}+\mathrm{H}^{*}$ & 0.52 & 1.30 & 0.78 \\
\hline 46 & $\mathrm{CH}_{3} \mathrm{CH}_{2} \mathrm{Cl}^{*}+* \rightarrow \mathrm{CH}_{3} \mathrm{CH}_{2} *+\mathrm{Cl}^{*}$ & -0.76 & 1.14 & 1.91 \\
\hline 47 & $\mathrm{ClCH}_{2} \mathrm{CH}_{2} \mathrm{Cl}^{*}+* \rightarrow \mathrm{ClCH}_{2} \mathrm{CH}_{2} *+\mathrm{Cl}^{*}$ & -1.08 & 1.07 & 2.15 \\
\hline 48 & $\mathrm{CH}_{3} \mathrm{CHCl}^{*}+* \rightarrow \mathrm{CH}_{3} \mathrm{CH}^{*}+\mathrm{Cl}^{*}$ & -0.77 & 0.18 & 0.96 \\
\hline 49 & $\mathrm{ClCH}_{2} \mathrm{CH}_{2} *+* \rightarrow \mathrm{CH}_{2} \mathrm{CH}_{2} *+\mathrm{Cl}^{*}$ & -1.08 & 0.33 & 1.41 \\
\hline 50 & $\mathrm{ClCH}_{2} \mathrm{CHCl}^{*}+* \rightarrow \mathrm{CH}_{2} \mathrm{CHCl}^{*}+\mathrm{Cl}^{*}$ & -1.05 & 0.71 & 1.76 \\
\hline
\end{tabular}




\begin{tabular}{|c|c|c|c|c|}
\hline 51 & $\mathrm{ClCH}_{2} \mathrm{CHCl}^{*}+* \rightarrow \mathrm{ClCH}_{2} \mathrm{CH}^{*}+\mathrm{Cl}^{*}$ & -0.89 & 0.22 & 1.11 \\
\hline 52 & $\mathrm{CH}_{2} \mathrm{CHCl}^{*}+* \rightarrow \mathrm{CH}_{2} \mathrm{CH}^{*}+\mathrm{Cl}^{*}$ & -0.60 & 0.35 & 0.95 \\
\hline 53 & $\mathrm{ClCHCHCl}^{*}+* \rightarrow \mathrm{ClCHCH}^{*}+\mathrm{Cl}^{*}$ & -0.69 & 0.37 & 1.06 \\
\hline 54 & $\mathrm{ClCH}_{2} \mathrm{CH}^{*}+* \rightarrow \mathrm{CH}_{2} \mathrm{CH}^{*}+\mathrm{Cl}^{*}$ & -0.76 & 0.51 & 1.26 \\
\hline 55 & $\mathrm{CH}_{3} \mathrm{CCl}^{*}+* \rightarrow \mathrm{CH}_{3} \mathrm{C}^{*}+\mathrm{Cl}^{*}$ & -1.09 & 0.04 & 1.13 \\
\hline 56 & $\mathrm{ClCH}_{2} \mathrm{CCl}^{*}+* \rightarrow \mathrm{CH}_{2} \mathrm{CCl}^{*}+\mathrm{Cl}^{*}$ & \multicolumn{3}{|c|}{ does not occurb } \\
\hline 57 & $\mathrm{ClCH}_{2} \mathrm{CCl}^{*}+* \rightarrow \mathrm{ClCH}_{2} \mathrm{C}^{*}+\mathrm{Cl}^{*}$ & -1.01 & 0.03 & 1.04 \\
\hline 58 & $\mathrm{ClCH}_{2} \mathrm{C}^{*}+* \rightarrow \mathrm{CH}_{2} \mathrm{C}^{*}+\mathrm{Cl}^{*}$ & -0.92 & 0.40 & 1.32 \\
\hline 59 & $\mathrm{ClCHCH}^{*}+* \rightarrow \mathrm{CHCH}^{*}+\mathrm{Cl}^{*}$ & -1.01 & 0.36 & 1.38 \\
\hline 60 & $\mathrm{CH}_{2} \mathrm{CCl}^{*}+* \rightarrow \mathrm{CH}_{2} \mathrm{C}^{*}+\mathrm{Cl}^{*}$ & -1.20 & 0.26 & 1.46 \\
\hline 61 & $\mathrm{ClCHCCl}^{*}+* \rightarrow \mathrm{CHCCl}^{*}+\mathrm{Cl}^{*}$ & -1.19 & 0.19 & 1.38 \\
\hline 62 & $\mathrm{ClCHCCl}^{*}+* \rightarrow \mathrm{ClCHC}^{*}+\mathrm{Cl}^{*}$ & -1.30 & 0.30 & 1.60 \\
\hline 63 & $\mathrm{ClCHC}^{*}+* \rightarrow \mathrm{CHC}^{*}+\mathrm{Cl}^{*}$ & -0.46 & 0.57 & 1.04 \\
\hline 64 & $\mathrm{CHCCl}^{*}+* \rightarrow \mathrm{CHC}^{*}+\mathrm{Cl}^{*}$ & -0.57 & 0.63 & 1.20 \\
\hline 65 & $\mathrm{ClCCCl}^{*}+* \rightarrow \mathrm{ClCC}^{*}+\mathrm{Cl}^{*}$ & -0.44 & 0.74 & 1.18 \\
\hline
\end{tabular}

${ }^{a}$ Both $\Delta E$ and $E_{a}$ are defined with respect to the best initial state (BIS) and the best final state (BFS), in which the reactants/products are located at their preferred adsorption sites. When multiple reactant/product species are present, they are considered at infinite separation (zero interaction energy). $\Delta E=E(\mathrm{BFS})-E(\mathrm{BIS}) ; E_{a}=E(\mathrm{TS})-E(\mathrm{BIS}) ; E_{a}$ rev $=E(\mathrm{TS})-E(\mathrm{BFS})$.

${ }^{\mathrm{b}}$ We were unable to determine any feasible reaction path from NEB calculations: another dechlorination step for the same reactant species (Step 48: $\mathrm{ClCH}_{2} \mathrm{CCl}^{*}+* \rightarrow \mathrm{ClCH}_{2} \mathrm{C}^{*}+\mathrm{Cl}^{*}$ ) would always spontaneously occur ahead of this step.

Table S2: Calculated Shomate parameters ${ }^{a}$ for gas-phase species in 1,2-DCA hydrodechlorination reaction network

\begin{tabular}{|l|r|r|r|r|r|r|r|r|}
\hline Species & \multicolumn{1}{l|l}{ A } & \multicolumn{1}{l|l}{ B } & \multicolumn{1}{l|}{ C } & \multicolumn{1}{l|}{ E } & F & G & \multicolumn{1}{l|}{ H } \\
\hline $\mathrm{CH}_{3} \mathrm{CH}_{3}$ & 5.9728 & 203.8759 & -99.8950 & 19.3316 & 0.3958 & -3729.83 & 202.54 & -3721.16 \\
\hline $\mathrm{CH}_{3} \mathrm{CH}_{2} \mathrm{Cl}$ & -1.0185 & 252.1535 & -159.9266 & 41.8067 & 0.1618 & -3597.57 & 208.19 & -3588.53 \\
\hline $\mathrm{ClCH}_{2} \mathrm{CH}_{2} \mathrm{Cl}$ & 16.9574 & 245.6316 & -166.6432 & 45.4878 & -0.2218 & -3476.39 & 258.06 & -3461.06 \\
\hline $\mathrm{CH}_{2} \mathrm{CH}_{2}$ & -2.3972 & 175.2570 & -106.9242 & 27.4315 & 0.1386 & -2950.28 & 175.13 & -2944.56 \\
\hline $\mathrm{CH}_{2} \mathrm{CHCl}$ & 19.9232 & 163.4255 & -112.8009 & 32.0568 & -0.2235 & -2849.68 & 249.61 & -2836.66 \\
\hline $\mathrm{ClCHCHCl}$ & 31.9180 & 167.2932 & -126.8948 & 37.8781 & -0.2519 & -2730.35 & 295.89 & -2713.59 \\
\hline $\mathrm{CHCH}$ & 42.3943 & 36.6811 & -14.3841 & 3.2434 & -0.7447 & -2151.77 & 241.77 & -2135.12 \\
\hline $\mathrm{CHCCl}$ & 55.3654 & 28.6695 & -11.1666 & 2.0159 & -0.7610 & -2023.33 & 300.58 & -2003.09 \\
\hline $\mathrm{ClCCCl}$ & 60.9155 & 36.5496 & -21.0437 & 4.6780 & -0.4189 & -1887.33 & 337.41 & -1866.31 \\
\hline $\mathrm{H}_{2}$ & 30.1727 & -3.2680 & 2.6602 & 0.2111 & -0.0322 & -626.99 & 171.61 & -618.01 \\
\hline $\mathrm{HCl}$ & 32.2263 & -13.9532 & 20.3353 & -7.1503 & -0.0510 & -571.56 & 232.89 & -562.23 \\
\hline
\end{tabular}

${ }^{a}$ The temperature dependence of the standard entropy and enthalpy of each species can be described using the Shomate equations (Equation S1) and the tabulated parameters[2]:

$$
\begin{aligned}
& t=T[\mathrm{~K}] / 1000 \\
& C_{P}[\mathrm{~J} / \mathrm{mol} \cdot \mathrm{K}]=A+B t+C t^{2}+D t^{3}+E / t \\
& \left(H^{\circ}-H^{\circ}{ }_{298.15 K}\right)[\mathrm{kJ} / \mathrm{mol}]=A t+B t^{2} / 2+C t^{3} / 3+D t^{4} / 4-E / t+F-H \\
& S^{\circ}[\mathrm{J} / \mathrm{mol} \cdot \mathrm{K}]=A \ln t+B t+C t^{2} / 2+D t^{3} / 3-E /\left(2 t^{2}\right)+G
\end{aligned}
$$


Table S3: Calculated Shomate parameters ${ }^{\mathrm{a}}$ for adsorbed species on $\mathrm{Pt}_{1} \mathrm{Cu}_{3}(111)$ in 1,2-DCA hydrodechlorination reaction network

\begin{tabular}{|c|c|c|c|c|c|c|c|c|}
\hline Species & $\mathbf{A}$ & B & $\mathrm{C}$ & D & $\mathbf{E}$ & $\mathbf{F}$ & G & H \\
\hline $\mathrm{CH}_{3} \mathrm{CH}_{3}$ & -3.3451 & 260.3326 & -151.7878 & 36.6780 & 0.5039 & -3746.66 & 37.63 & -3739.04 \\
\hline $\mathrm{CH}_{3} \mathrm{CH}_{2} \mathrm{Cl}$ & 15.2648 & 254.5526 & -162.7156 & 42.8026 & 0.0527 & -3664.27 & 68.39 & -3649.93 \\
\hline $\mathrm{ClCH}_{2} \mathrm{CH}_{2} \mathrm{Cl}$ & 29.2481 & 257.9369 & -179.1956 & 49.9430 & -0.2321 & -3546.16 & 103.08 & -3526.68 \\
\hline $\mathrm{CH}_{3} \mathrm{CH}_{2}$ & 2.2618 & 230.9509 & -143.7682 & 37.2223 & -0.1109 & -3397.32 & 8.73 & -3387.21 \\
\hline $\mathrm{CH}_{3} \mathrm{CHCl}$ & 24.7303 & 212.8501 & -140.6303 & 38.0704 & -0.3233 & -3316.69 & 55.51 & -3299.94 \\
\hline $\mathrm{ClCH}_{2} \mathrm{CH}_{2}$ & 19.1103 & 229.0985 & -156.7059 & 43.5082 & -0.5147 & -3304.85 & 40.30 & -3288.55 \\
\hline $\mathrm{ClCH}_{2} \mathrm{CHCl}$ & 39.9650 & 213.3710 & -154.6358 & 44.4529 & -0.6389 & -3203.94 & 98.15 & -3181.68 \\
\hline $\mathrm{CH}_{2} \mathrm{CH}_{2}$ & -0.1344 & 198.9921 & -133.6846 & 37.2274 & -0.4624 & -3063.62 & -17.12 & -3054.38 \\
\hline $\mathrm{CH}_{2} \mathrm{CHCl}$ & 31.1018 & 179.3849 & -127.8948 & 36.8692 & -0.7467 & -2953.99 & 63.29 & -2935.29 \\
\hline $\mathrm{ClCHCHCl}$ & 53.9850 & 155.9331 & -115.4557 & 33.4824 & -0.9704 & -2838.67 & 123.35 & -2813.35 \\
\hline $\mathrm{CH}_{3} \mathrm{CH}$ & 12.1504 & 186.2341 & -116.5273 & 30.1992 & -0.3030 & -3023.08 & 20.02 & -3011.14 \\
\hline $\mathrm{ClCH}_{2} \mathrm{CH}$ & 31.3500 & 179.5066 & -125.6031 & 35.3810 & -0.7909 & -2918.27 & 53.71 & -2899.33 \\
\hline $\mathrm{CH}_{3} \mathrm{CCl}$ & 37.1045 & 159.2501 & -104.4774 & 28.0837 & -0.4154 & -2933.87 & 78.40 & -2915.20 \\
\hline $\mathrm{ClCH}_{2} \mathrm{CCl}$ & 63.3283 & 126.4270 & -81.9835 & 20.7719 & -1.0670 & -2826.41 & 135.00 & -2799.01 \\
\hline $\mathrm{CH}_{3} \mathrm{C}$ & 13.8847 & 152.3643 & -96.6514 & 25.2307 & -0.2552 & -2695.22 & 20.49 & -2684.26 \\
\hline $\mathrm{ClCH}_{2} \mathrm{C}$ & 30.1088 & 152.3196 & -111.2900 & 32.0680 & -0.6433 & -2580.46 & 51.71 & -2563.48 \\
\hline $\mathrm{CH}_{2} \mathrm{CH}$ & 19.3102 & 148.2234 & -97.9161 & 26.7105 & -0.6030 & -2675.03 & 21.16 & -2661.47 \\
\hline $\mathrm{ClCHCH}$ & 49.3432 & 99.9754 & -58.4978 & 13.3575 & -1.1405 & -2580.88 & 99.09 & -2558.39 \\
\hline $\mathrm{CH}_{2} \mathrm{CCl}$ & 52.9376 & 95.3151 & -57.1537 & 13.7402 & -1.0437 & -2583.77 & 99.39 & -2560.73 \\
\hline $\mathrm{ClCHCCl}$ & 60.6391 & 111.2710 & -85.5998 & 25.3782 & -0.8027 & -2479.30 & 139.67 & -2454.29 \\
\hline $\mathrm{CH}_{2} \mathrm{C}$ & 38.4689 & 67.9146 & -31.0217 & 5.0404 & -0.8077 & -2342.83 & 62.32 & -2325.90 \\
\hline $\mathrm{ClCHC}$ & 46.8861 & 80.2476 & -53.2527 & 13.6393 & -0.8821 & -2242.83 & 85.83 & -2222.76 \\
\hline $\mathrm{CHCH}$ & 23.6747 & 117.8529 & -85.7833 & 25.1332 & -0.9162 & -2362.65 & 20.45 & -2347.99 \\
\hline $\mathrm{CHCCl}$ & 49.7630 & 79.5013 & -55.3593 & 14.8074 & -1.1339 & -2269.92 & 83.22 & -2248.21 \\
\hline $\mathrm{ClCCCl}$ & 59.5940 & 93.8957 & -84.9608 & 27.5611 & -0.7490 & -2153.51 & 128.20 & -2129.75 \\
\hline $\mathrm{CHC}$ & 37.1208 & 50.9742 & -31.4367 & 7.8056 & -0.9462 & -1982.23 & 52.63 & -1965.99 \\
\hline $\mathrm{ClCC}$ & 44.6448 & 63.9617 & -53.7693 & 16.5083 & -0.5458 & -1856.49 & 83.63 & -1838.94 \\
\hline $\mathrm{H}$ & 10.0406 & 10.6950 & 10.8935 & $\begin{array}{l}-9.5681 \\
\end{array}$ & -0.7658 & -365.51 & 6.53 & -359.39 \\
\hline $\mathrm{Cl}$ & 17.0393 & 25.1498 & -28.4790 & 10.9195 & 0.1583 & -363.42 & 49.71 & -357.99 \\
\hline
\end{tabular}

a The temperature dependence of the standard entropy and enthalpy of each species can be described using the Shomate equations (Equation S1) and the tabulated parameters [2].

Table S4: Calculated Shomate parameters ${ }^{\mathrm{a}}$ for transition states of surface elementary steps $\mathrm{on} \mathrm{Pt}_{1} \mathrm{Cu}_{3}(111)$ in 1,2DCA hydrodechlorination reaction network

\begin{tabular}{|l|l|l|l|l|l|l|l|l|}
\hline Rxn $\#^{\mathrm{b}}$ & $\mathbf{A}$ & $\mathbf{B}$ & $\mathbf{C}$ & $\mathbf{D}$ & $\mathbf{E}$ & $\mathbf{F}$ & $\mathbf{G}$ & $\mathbf{H}$ \\
\hline 12 & -7.9240 & 248.5808 & -146.5991 & 35.6096 & 0.1901 & -3677.54 & -16.53 & -3670.72 \\
\hline 13 & 9.3632 & 246.4183 & -159.1761 & 41.7223 & -0.1836 & -3576.88 & 21.71 & -3563.84 \\
\hline 14 & 14.6428 & 234.7290 & -149.3647 & 38.7251 & -0.1389 & -3573.30 & 38.44 & -3559.28 \\
\hline 15 & 39.9679 & 229.4750 & -156.9341 & 42.7867 & -0.4965 & -3462.91 & 99.35 & -3440.43 \\
\hline 16 & -1.8161 & 251.1160 & -173.7667 & 48.5011 & -0.3104 & -3346.95 & -12.81 & -3336.72 \\
\hline 17 & 9.1739 & 211.1139 & -128.8041 & 32.2517 & -0.2064 & -3321.07 & 8.01 & -3309.33 \\
\hline 18 & 22.8427 & 226.2683 & -162.8190 & 46.4184 & -0.5283 & -3227.93 & 40.95 & -3210.64 \\
\hline 19 & 33.4733 & 186.8054 & -118.7557 & 30.5788 & -0.3881 & -3215.58 & 70.46 & -3196.98 \\
\hline 20 & 21.0930 & 233.5553 & -170.9272 & 49.3265 & -0.6713 & -3228.55 & 38.64 & -3211.04 \\
\hline 21 & 28.6164 & 203.1050 & -136.3282 & 36.8688 & -0.6803 & -3214.47 & 48.82 & -3195.76 \\
\hline 22 & 43.4796 & 213.8205 & -164.2813 & 48.5313 & -0.7747 & -3108.49 & 95.40 & -3084.78 \\
\hline 23 & 52.2652 & 178.4022 & -124.7309 & 34.4375 & -0.7507 & -3102.50 & 115.13 & -3077.51 \\
\hline 24 & 18.2079 & 167.7448 & -105.2258 & 27.1527 & -0.3457 & -2958.42 & 31.30 & -2945.25 \\
\hline
\end{tabular}




\begin{tabular}{|c|c|c|c|c|c|c|c|c|}
\hline 25 & 53.2834 & 110.1188 & -56.7849 & 10.5021 & -1.0834 & -2868.88 & 105.93 & $\begin{array}{l}-2844.94 \\
\end{array}$ \\
\hline 26 & 33.1814 & 167.7888 & -117.2041 & 32.4239 & -0.4586 & -2865.58 & 72.46 & -2847.66 \\
\hline 27 & 62.6454 & 124.9988 & -84.6690 & 21.8506 & -0.9487 & -2768.91 & 141.33 & -2742.20 \\
\hline 28 & 9.7039 & 204.8482 & -145.5132 & 41.1644 & -0.6666 & -2965.61 & -9.05 & -2952.58 \\
\hline 29 & 9.7948 & 196.1092 & -132.8562 & 36.0902 & -0.2278 & -2950.28 & 17.70 & -2938.98 \\
\hline 30 & 36.2613 & 177.6815 & -134.0191 & 39.2812 & -0.9732 & -2832.35 & 55.07 & -2811.48 \\
\hline 31 & 32.0788 & 170.0463 & -118.6445 & 32.7571 & -0.6249 & -2849.81 & 58.63 & -2831.58 \\
\hline 32 & 38.4244 & 172.8282 & -130.9034 & 38.6467 & -0.9003 & -2839.93 & 70.88 & -2818.86 \\
\hline 33 & 52.1960 & 173.2555 & -142.3416 & 43.6275 & -0.9629 & -2729.95 & 112.01 & -2704.63 \\
\hline 34 & 10.1239 & 175.1161 & -129.1124 & 37.1751 & -0.6290 & -2609.28 & -8.99 & -2597.44 \\
\hline 35 & 29.2024 & 163.0887 & -129.5898 & 38.7316 & -0.7765 & -2493.65 & 39.05 & -2476.16 \\
\hline 36 & 24.1167 & 149.7398 & -111.4057 & 32.7031 & -0.9200 & -2569.60 & 24.07 & -2553.59 \\
\hline 37 & 23.8966 & 129.1106 & -81.9015 & 21.1173 & -0.6210 & -2627.58 & 26.28 & -2613.31 \\
\hline 38 & 32.0331 & 162.3329 & -132.1261 & 40.2256 & -0.9014 & -2536.95 & 43.87 & -2518.25 \\
\hline 39 & 49.5809 & 95.4385 & -55.0148 & 11.2029 & -0.9564 & -2518.30 & 89.68 & -2496.53 \\
\hline 40 & 32.6612 & 157.9986 & -126.8701 & 38.2793 & -0.9000 & -2549.00 & 44.98 & -2530.26 \\
\hline 41 & 61.9333 & 112.7355 & -90.7167 & 26.3288 & -0.9821 & -2392.32 & 131.97 & -2366.30 \\
\hline 42 & 23.0589 & 121.1158 & -93.6541 & 27.6848 & -0.7689 & -2255.54 & 20.75 & -2241.47 \\
\hline 43 & 48.2194 & 82.7280 & -61.1545 & 16.1715 & -0.8505 & -2130.38 & 87.57 & -2109.98 \\
\hline 44 & 40.2257 & 62.1310 & -29.6581 & 4.6664 & -0.9036 & -2259.81 & 59.17 & -2242.28 \\
\hline 45 & 47.7333 & 75.2076 & -52.1624 & 13.4647 & -0.4816 & -2138.27 & 90.90 & -2119.52 \\
\hline 46 & 25.0313 & 202.8105 & -122.6880 & 31.4550 & -0.1755 & -3564.40 & 76.31 & -3548.36 \\
\hline 47 & 45.5278 & 190.5095 & -124.9198 & 34.0110 & -0.4893 & -3455.40 & 128.48 & -3432.75 \\
\hline 48 & 25.3718 & 195.5357 & -127.2836 & 34.2955 & -0.3642 & -3301.39 & 58.25 & -3284.97 \\
\hline 49 & 20.9461 & 208.5350 & -141.2729 & 39.3322 & -0.4556 & -3280.00 & 52.86 & -3264.13 \\
\hline 50 & 53.6170 & 165.3603 & -114.1081 & 31.9617 & -0.8656 & -3166.20 & 123.04 & -3140.90 \\
\hline 51 & 41.7802 & 192.2696 & -137.7178 & 39.5371 & -0.6677 & -3181.08 & 102.62 & -3158.97 \\
\hline 52 & 50.6478 & 110.3598 & -61.4229 & 14.0253 & -1.0385 & -2893.60 & 112.36 & -2870.62 \\
\hline 53 & 59.6903 & 123.9053 & -86.9818 & 24.3408 & -0.9987 & -2788.83 & 143.04 & -2762.90 \\
\hline 54 & 31.5895 & 161.4080 & -111.6313 & 31.5098 & -0.7107 & -2899.94 & 56.96 & -2881.89 \\
\hline 55 & 31.3165 & 154.4486 & -100.8576 & 27.0749 & -0.3321 & -2927.36 & 66.93 & -2910.88 \\
\hline 56 & 63.1333 & 130.7663 & -89.4318 & 24.1855 & -0.9900 & -2802.22 & 148.72 & -2775.01 \\
\hline 57 & 57.2352 & 120.3563 & -76.0197 & 18.7082 & -0.9678 & -2818.23 & 125.74 & -2793.20 \\
\hline 58 & 46.7895 & 89.1673 & -49.8056 & 10.6914 & -1.0633 & -2564.10 & 83.06 & -2543.04 \\
\hline 59 & 56.0415 & 85.2570 & -63.5447 & 20.3747 & -0.7401 & -2530.52 & 121.93 & -2508.06 \\
\hline 60 & 48.8157 & 87.3904 & -51.9692 & 12.6524 & -0.8694 & -2570.11 & 93.53 & -2549.19 \\
\hline 61 & 80.3521 & 48.2589 & -31.7373 & 8.8368 & -0.9177 & -2397.37 & 181.73 & -2368.46 \\
\hline 62 & 57.4683 & 103.4828 & -81.1988 & 24.6141 & -0.6131 & -2448.34 & 133.70 & -2425.21 \\
\hline 63 & 47.3653 & 69.0514 & -51.1882 & 15.2288 & -0.7467 & -2198.36 & 86.25 & -2179.09 \\
\hline 64 & 53.3955 & 76.9978 & -60.0026 & 18.5642 & -0.7221 & -2207.36 & 102.23 & -2186.09 \\
\hline 65 & 54.6673 & 85.2157 & -77.4148 & 25.3762 & -0.4973 & -2085.60 & 122.21 & -2064.48 \\
\hline
\end{tabular}

${ }^{a}$ The temperature dependence of the standard entropy and enthalpy of each species can be described using the Shomate equations (Equation S1) and the tabulated parameters [2].

${ }^{\mathrm{b}}$ The reaction numbers are the same as that in Scheme S1. 


\section{References}

[1] L. Xu, E.E. Stangland, J.A. Dumesic, M. Mavrikakis, Hydrodechlorination of 1,2Dichloroethane on Platinum Catalysts: Insights from Reaction Kinetics Experiments, DFT, and Microkinetic Modeling, ACS Catal. 11 (2021) 7890-7905.

[2] P.J. Linstrom, W.G. Mallard, eds., NIST Chemistry WebBook, NIST Standard Reference Database Number 69, National Institute of Standards and Technology, Gaithersburg MD, 20899, 2017. https://doi.org/10.18434/T4D303. 